\title{
The role of meteorological conditions and pollution control strategies in reducing air pollution in Beijing during APEC 2014 and Victory Parade 2015
}

\section{Pengfei Liang et al.}

Correspondence to: Tong Zhu (tzhu@pku.edu.cn)

The copyright of individual parts of the supplement might differ from the CC BY 3.0 License. 
14 S1. Temperature steps and duration of NOISH thermal-optical method protocol.

15 Table S1. Temperature steps and duration of NOISH thermal-optical method protocol.

\begin{tabular}{ccc}
\hline Carrier gas & Duration $(\mathrm{sec})$ & Temperature $\left({ }^{\circ} \mathrm{C}\right)$ \\
\hline $\mathrm{He}-1$ & 10 & Ambient \\
$\mathrm{He}-2$ & 95 & 600 \\
$\mathrm{He}-3$ & 90 & 840 \\
$\mathrm{He}-4$ & 30 & No heat \\
$\mathrm{O}_{2}-1$ & 35 & 550 \\
$\mathrm{O}_{2}-2$ & 45 & 650 \\
$\mathrm{O}_{2}-3$ & 115 & 870 \\
$\mathrm{CalGas}$ & 125 & No heat \\
\hline
\end{tabular}

16

17 

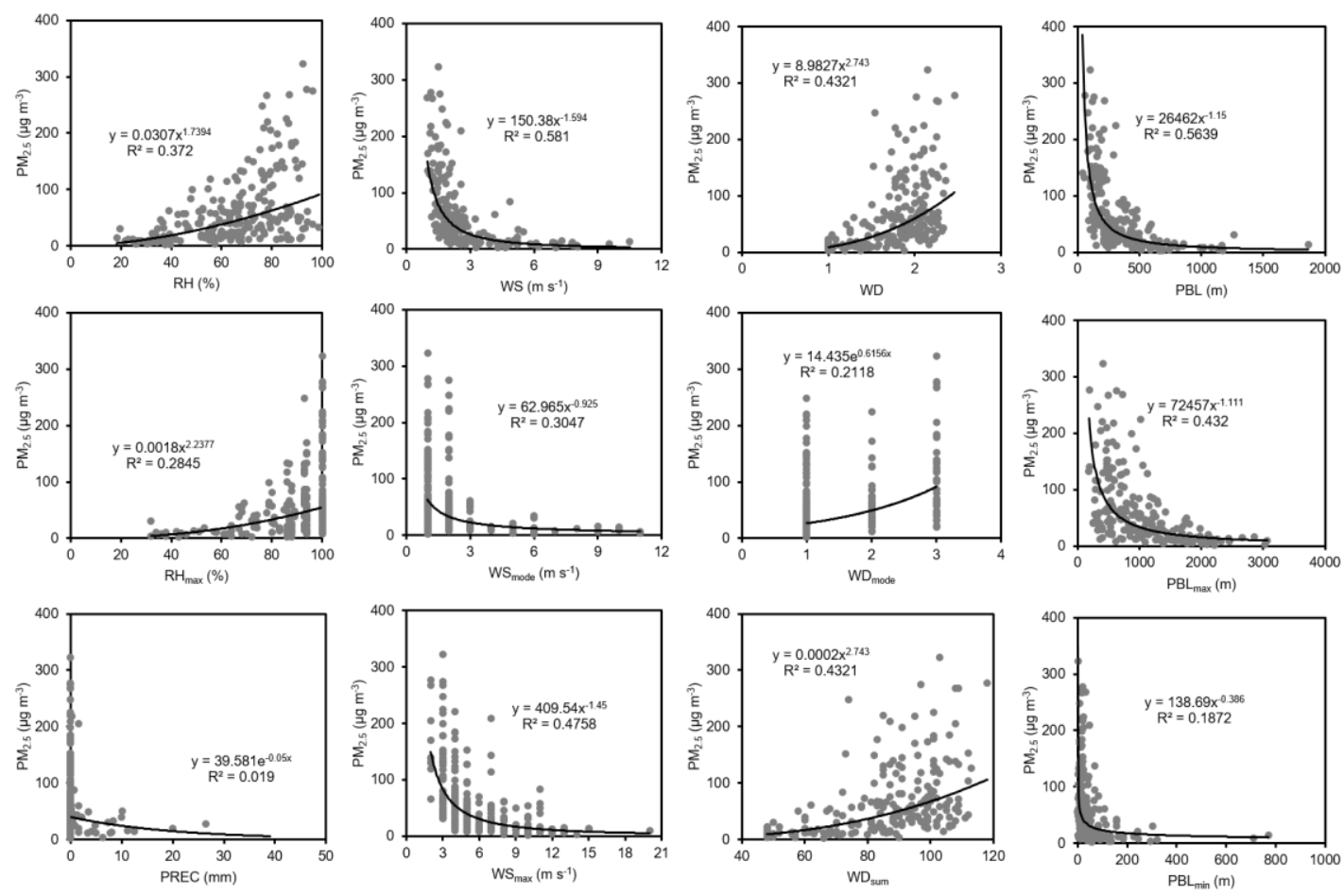

Fig S2. Scatter plot and correlation between $\mathrm{PM}_{2.5}$ concentrations ( $y$ axis) and meteorological parameters ( $x$ axis) during the APEC and Parade sampling periods. 


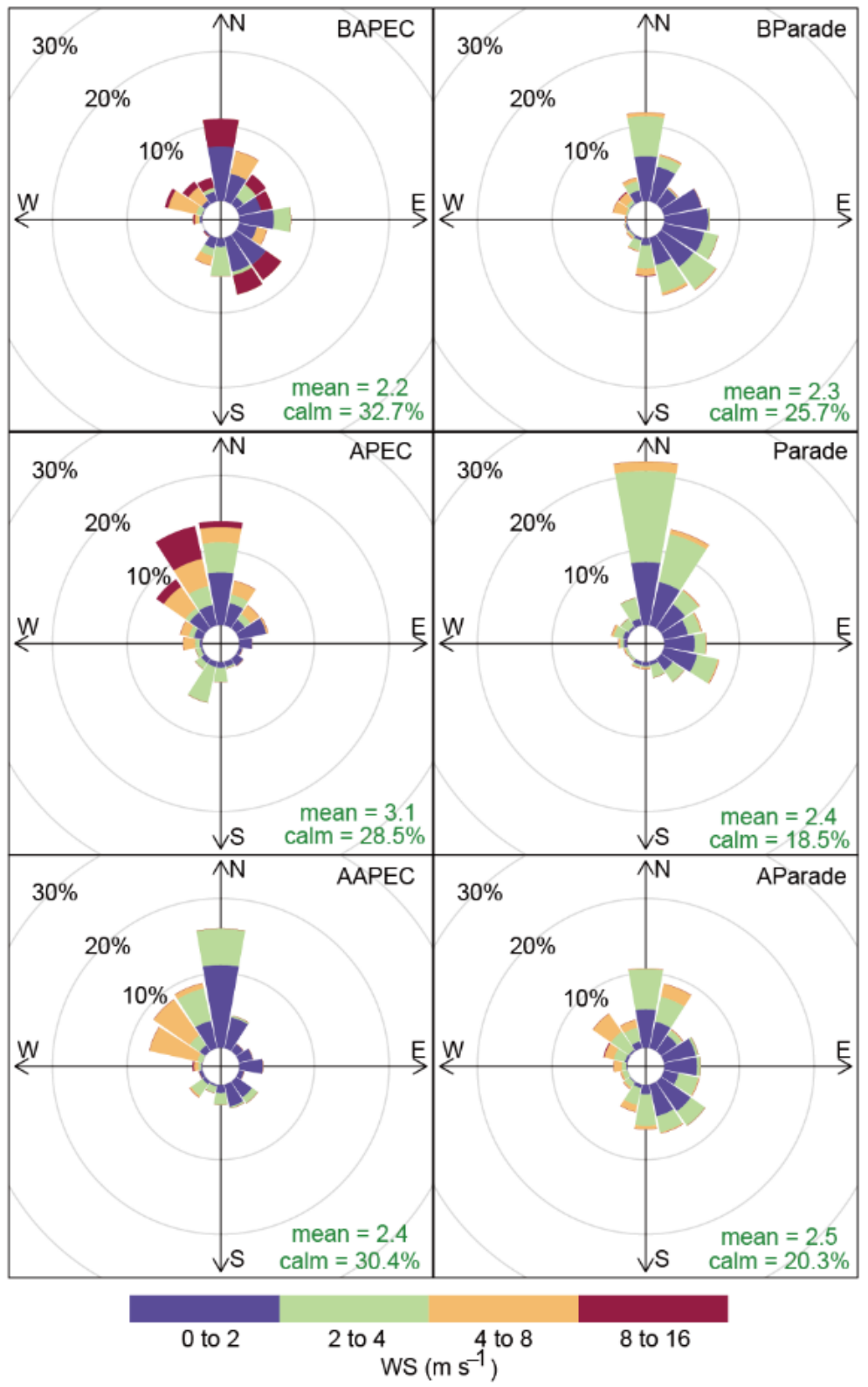

Figure S3. Wind rose plots based on frequencies of half-hourly data before APEC (BAPEC), during APEC, and after APEC (AAPEC) on the left, and before Parade (BParade), during Parade, and after Parade (AParade) on the right.

Figure S3 shows the prevalence of WD during the APEC and Parade campaigns.

During APEC, the prevailing WD was from the north and northwest, and accounted for 
$31 \quad 30-40 \%$ of the wind frequency. The mean WS during APEC was $3.1 \mathrm{~m} \mathrm{~s}^{-1}$, higher than

32 before APEC $\left(2.2 \mathrm{~m} \mathrm{~s}^{-1}\right)$ and after APEC $\left(2.4 \mathrm{~m} \mathrm{~s}^{-1}\right)$. The "calm and variable" 33 proportion of APEC was $28.5 \%$, which was lowest during the APEC campaign. During

34 Parade, a northern and northeastern WD accounted for more than $30 \%$ of the wind 35 frequency, and the "calm and variable" proportion was $18.5 \%$, much lower than before 36 Parade (25.7\%) and after Parade (20.3\%).

37 


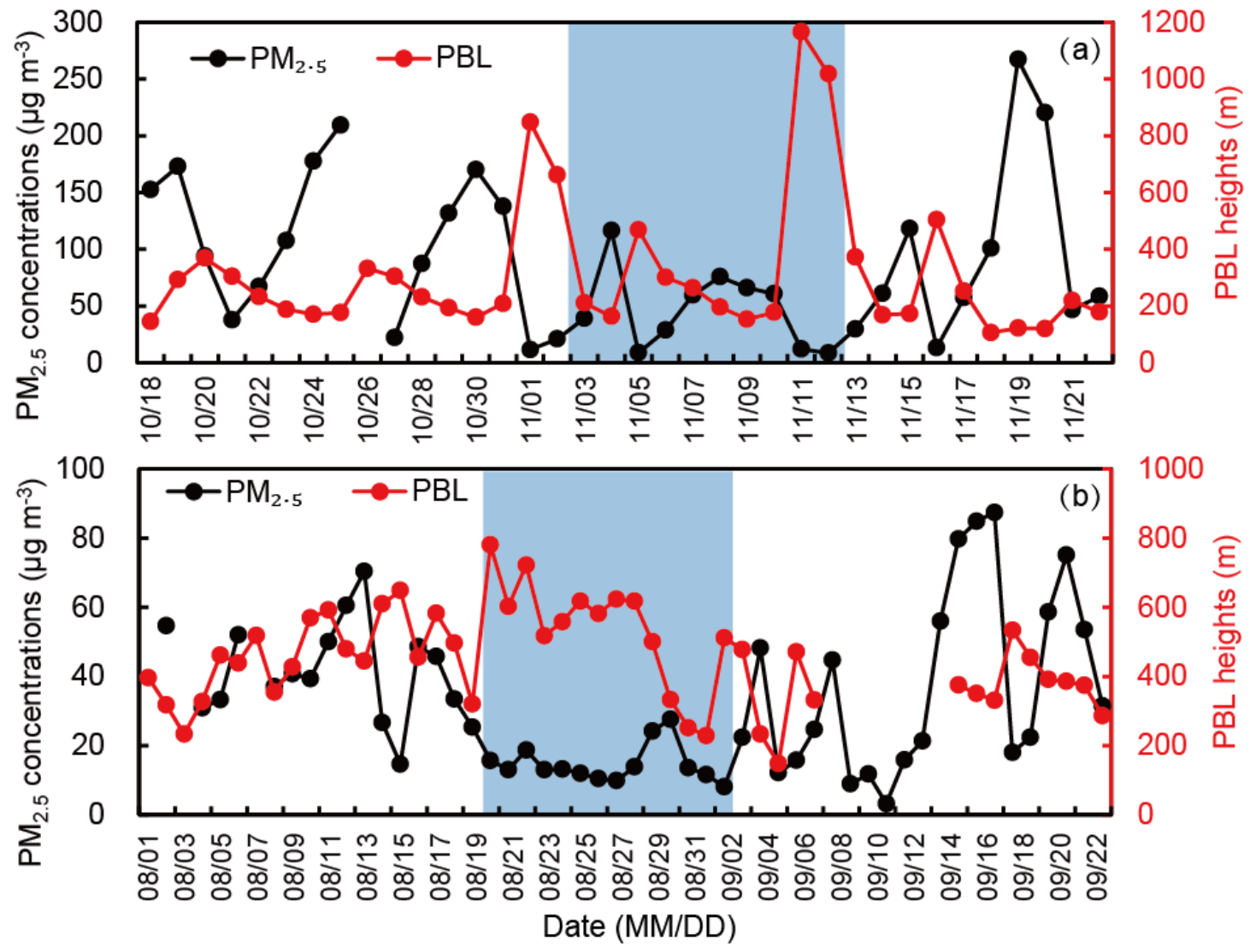

Figure $\mathrm{S} 4$. Time series of daily $\mathrm{PM}_{2.5}$ concentrations and planetary boundary layer (PBL) heights during the (a) APEC and (b) Parade campaigns. The black line represents PM$_{2.5}$ concentrations and the red line represents PBL heights. The blue-shaded areas highlight the pollution control periods of APEC 2014 (3 November to 12 November 2014) and Parade 2015 (20 August to 3 September 2015).

Figure S4 shows a time series of daily average $\mathrm{PM}_{2.5}$ concentrations and $\mathrm{PBL}$ heights during the APEC and Parade campaigns, indicating that they have an anticorrelation. In both periods of before and after APEC, the PBL heights were mostly less than $400 \mathrm{~m}$. Compared with during APEC and during Parade, the PBL heights increased on 5, 11, and 12 November during APEC and were mostly higher than $400 \mathrm{~m}$ during Parade, which was much more favourable for the diffusion of air pollutants during the 
control period.

54

55 
S5. Statistical summary showing the meteorological conditions and the concentrations of pollutants on the days with stable meteorological conditions.

Table S5. Statistical summary showing the meteorological conditions (WS and PBL height), and the concentrations of pollutants on the days with stable meteorological conditions during the APEC campaign. BAPEC/BParade: before APEC/Parade, AAPEC/AParade: after APEC/Parade.

\begin{tabular}{|c|c|c|c|c|c|c|c|c|c|c|c|c|c|}
\hline & WS & PBL & $\mathrm{PM}_{2.5}$ & $\mathrm{OC}$ & $\mathrm{EC}$ & $\mathrm{SO}_{4}{ }^{2-}$ & $\mathrm{NO}_{3}^{-}$ & $\mathrm{NH}_{4}^{+}$ & $\mathrm{SO}_{2}$ & NO & $\mathrm{NO}_{\mathrm{x}}$ & $\mathrm{O}_{3}$ \\
\hline & & $\left(\mathrm{m} \mathrm{s}^{-1}\right)$ & (m) & \multicolumn{10}{|c|}{$\left(\mu \mathrm{g} \mathrm{m}^{-3}\right)$} \\
\hline \multirow{9}{*}{ BAPEC } & $10 / 18$ & 1.27 & 145 & 153 & - & - & 14.3 & 43.0 & 19.4 & - & 73 & 240 & 45.2 \\
\hline & $10 / 22$ & 1.46 & 233 & 67 & 10.9 & 3.6 & 7.4 & 14.0 & 9.3 & 12.1 & 59 & 153 & 4.4 \\
\hline & $10 / 23$ & 1.46 & 188 & 108 & 18.0 & 2.6 & 11.3 & 23.7 & 13.2 & 11.5 & 91 & 200 & 6.6 \\
\hline & $10 / 24$ & 1.52 & 171 & 177 & 20.5 & 3.0 & 24.6 & 54.2 & 28.3 & 12.0 & 73 & 205 & 20.4 \\
\hline & $10 / 28$ & 1.71 & 232 & 87 & 15.9 & 3.8 & 7.0 & 17.8 & 7.9 & 13.0 & 69 & 165 & 14.2 \\
\hline & $10 / 29$ & 1.10 & 193 & 132 & 23.6 & 5.3 & 10.6 & 35.2 & 14.0 & 15.6 & 99 & 229 & 10.0 \\
\hline & $10 / 30$ & 1.00 & 160 & 170 & 26.0 & 4.0 & 18.5 & 56.0 & 25.7 & 18.4 & 77 & 195 & 7.5 \\
\hline & $10 / 31$ & 1.50 & 209 & 138 & 17.0 & 3.9 & 13.5 & 29.3 & 16.1 & 8.1 & 79 & 183 & 4.6 \\
\hline & Mean & 1.38 & 191 & 129 & 18.8 & 3.7 & 13.4 & 34.2 & 16.7 & 12.9 & 77 & 196 & 14.1 \\
\hline \multirow{7}{*}{ APEC } & $11 / 3$ & 1.98 & 211 & 39 & 11.1 & 1.8 & 1.8 & 4.9 & 2.6 & 5.8 & 19 & 84 & 36.5 \\
\hline & $11 / 4$ & 1.85 & 163 & 116 & 22.7 & 2.9 & 9.6 & 33.1 & 13.2 & 26.0 & 31 & 144 & 20.5 \\
\hline & $11 / 7$ & 1.63 & 264 & 59 & 12.5 & 2.8 & 4.3 & 10.9 & 6.1 & 13.6 & 30 & 101 & 15.0 \\
\hline & $11 / 8$ & 2.00 & 196 & 76 & 17.3 & 2.4 & 7.3 & 21.1 & 8.8 & 11.8 & 26 & 101 & 33.6 \\
\hline & $11 / 9$ & 1.79 & 154 & 66 & 17.6 & 2.5 & 4.9 & 14.0 & 6.3 & 9.2 & 44 & 125 & 27.7 \\
\hline & $11 / 10$ & 2.13 & 177 & 61 & 14.3 & 1.8 & 5.6 & 17.9 & 7.5 & 10.2 & 31 & 115 & 29.2 \\
\hline & Mean & 1.90 & 194 & 70 & 15.9 & 2.4 & 5.6 & 17.0 & 7.4 & 12.7 & 30 & 112 & 27.1 \\
\hline \multirow{8}{*}{ AAPEC } & $11 / 14$ & 1.58 & 169 & 61 & 15.2 & 4.5 & 3.4 & 6.4 & 4.1 & 30.3 & 87 & 171 & 14.7 \\
\hline & $11 / 15$ & 1.38 & 173 & 118 & 24.2 & 6.6 & 7.2 & 19.6 & 10.6 & 52.0 & 148 & 276 & 5.0 \\
\hline & $11 / 17$ & 2.48 & 252 & 57 & 14.5 & 3.8 & 2.8 & 4.0 & 3.7 & 30.4 & 125 & 206 & 25.0 \\
\hline & $11 / 18$ & 1.44 & 106 & 101 & 27.1 & 3.8 & 6.3 & 14.3 & 8.3 & 54.5 & 162 & 285 & 6.2 \\
\hline & $11 / 19$ & 1.23 & 121 & 267 & 53.2 & 5.0 & 38.2 & 55.6 & 35.2 & 54.6 & 190 & 369 & 1.0 \\
\hline & $11 / 20$ & 1.94 & 120 & 220 & 41.6 & 3.7 & 26.2 & 46.9 & 28.8 & 38.8 & 200 & 383 & 2.9 \\
\hline & $11 / 22$ & 1.96 & 178 & 58 & 14.0 & 3.3 & 3.3 & 5.4 & 4.5 & 32.2 & 89 & 183 & 26.7 \\
\hline & Mean & 1.72 & 160 & 126 & 27.1 & 4.4 & 12.5 & 21.7 & 13.6 & 41.8 & 143 & 268 & 11.6 \\
\hline
\end{tabular}

63 The days with stable meteorological conditions were determined with the method

64 introduced in Section 3.2.1. As a result, eight days before APEC (18, 22, 23, 24, 28, 29,

6530 , and 31 October 2014), six days during $\operatorname{APEC}(3,4,7,8$, 9, and 10 November 2014),

66 and seven days after APEC (14, 15, 17, 18, 19, 20, and 22 November 2014) were 
defined as having stable meteorological conditions. Table S5 lists the meteorological conditions (WSs and PBL heights), and the concentrations of pollutants on the days with stable meteorological conditions during the APEC campaign. For the Parade campaign, only one day in each of the BParade, Parade, and AParade periods was defined as having stable meteorological conditions. This was considered to not be well representative of the Parade campaign. Thus, we only assessed the variation of air pollutant concentrations during stable meteorological periods of the APEC campaign. For days with stable meteorological conditions during the APEC campaign, the average WS was $1.4,1.9$, and $1.7 \mathrm{~m} \mathrm{~s}^{-1}$ before, during, and after APEC, respectively; and the average PBL height was 191, 194, and $160 \mathrm{~m}$ in the same three periods, respectively. This clearly shows that the meteorological conditions of days considered to be stable throughout the APEC campaign were very similar. 
$83 \bar{N}=f\left(\overline{x_{1}}, \overline{x_{2}}, \overline{x_{3}}\right)$

$84 S_{N}=\sqrt{\left(\frac{\partial f}{\partial x_{1}}\right)^{2} S_{x_{1}}{ }^{2}+\left(\frac{\partial f}{\partial x_{2}}\right)^{2} S_{x_{2}}{ }^{2}+\left(\frac{\partial f}{\partial x_{3}}\right)^{2} S_{x_{3}}{ }^{2}}$

85

In this study, $N$ represents the percentage reduction calculated by comparing the decreased average pollutant concentration during APEC to that before APEC, and $x$ represents the pollutant concentrations during and before APEC.

89 
S7. The percentage differences for the PM2.5 concentrations of four periods that were randomly selected from within the non-control stable days of the APEC 2014 and Parade 2015 campaigns.

Table S7. The percentage differences (PD) for the $\mathrm{PM}_{2.5}$ concentrations of four periods (P1, P2, P3, and P4) that were randomly selected from within the non-control stable days of the APEC 2014 and Parade 2015 campaigns.

\begin{tabular}{|c|c|c|c|c|c|c|c|c|c|}
\hline \multirow{2}{*}{ Periods } & \multirow{2}{*}{$\begin{array}{c}\text { Mean } \\
\text { values } \\
\left(\mu \mathrm{g} \mathrm{m}^{-3}\right)\end{array}$} & \multirow{2}{*}{$\mathrm{SD}\left(\mu \mathrm{g} \mathrm{m}^{-3}\right)$} & \multirow{2}{*}{$\begin{array}{l}\text { Total SD } \\
\left(\mu \mathrm{g} \mathrm{m}^{-3}\right)\end{array}$} & \multicolumn{4}{|c|}{ Percentage differences (PD)* } & \multirow{2}{*}{$\begin{array}{c}\text { Mean } \\
\text { PD }\end{array}$} & \multirow{2}{*}{$\begin{array}{l}\text { RMSE } \\
\text { of PD }\end{array}$} \\
\hline & & & & $\mathrm{P} 1$ & $\mathrm{P} 2$ & P3 & $\mathrm{P} 4$ & & \\
\hline $\mathrm{P} 1$ & 120 & 97 & \multirow{4}{*}{59} & - & - & - & - & \multirow{4}{*}{$-16 \%$} & \multirow{4}{*}{$18 \%$} \\
\hline $\mathrm{P} 2$ & 101 & 58 & & $-16 \%$ & - & - & - & & \\
\hline P3 & 96 & 40 & & $-20 \%$ & $-5 \%$ & - & - & & \\
\hline P4 & 87 & 23 & & $-28 \%$ & $-14 \%$ & $-9 \%$ & - & & \\
\hline
\end{tabular}

* Percentage difference $(\mathrm{PD})=\left(\right.$ Mean value of $\mathrm{P}_{\mathrm{n}+1}-$ Mean value of $\left.\mathrm{P}_{\mathrm{n}}\right) /$ Mean value of $\mathrm{P}_{\mathrm{n}} \times 100 \%$.

Table S7 lists the percentage differences among the mean $\mathrm{PM}_{2.5}$ concentrations of four periods that were randomly selected from within the non-control days of the APEC and Parade campaigns. Based on the assumptions that days with stable meteorological conditions were representative of the corresponding periods during the APEC campaign, and the emission intensities were constant, the percentage differences in the mean $\mathrm{PM}_{2.5}$ concentrations between these four random periods should be close to zero. The mean concentrations during $\mathrm{P} 1, \mathrm{P} 2, \mathrm{P} 3$, and $\mathrm{P} 4$ were $120,101,96$, and $87 \mu \mathrm{g} \mathrm{m}^{-3}$, respectively. The standard deviation (SD) during P1, P2, P3, and P4 were 97, 58, 40, and $23 \mu \mathrm{g} \mathrm{m}^{-3}$, respectively, with the average SD being $59 \mu \mathrm{g} \mathrm{m}^{-3}$. The mean value of the percentage differences of the mean $\mathrm{PM}_{2.5}$ concentrations between $\mathrm{P} 1, \mathrm{P} 2, \mathrm{P} 3$, and $\mathrm{P} 4$ was $-16 \%$, with a root mean square error (RMSE) of $18 \%$. 
Stable days
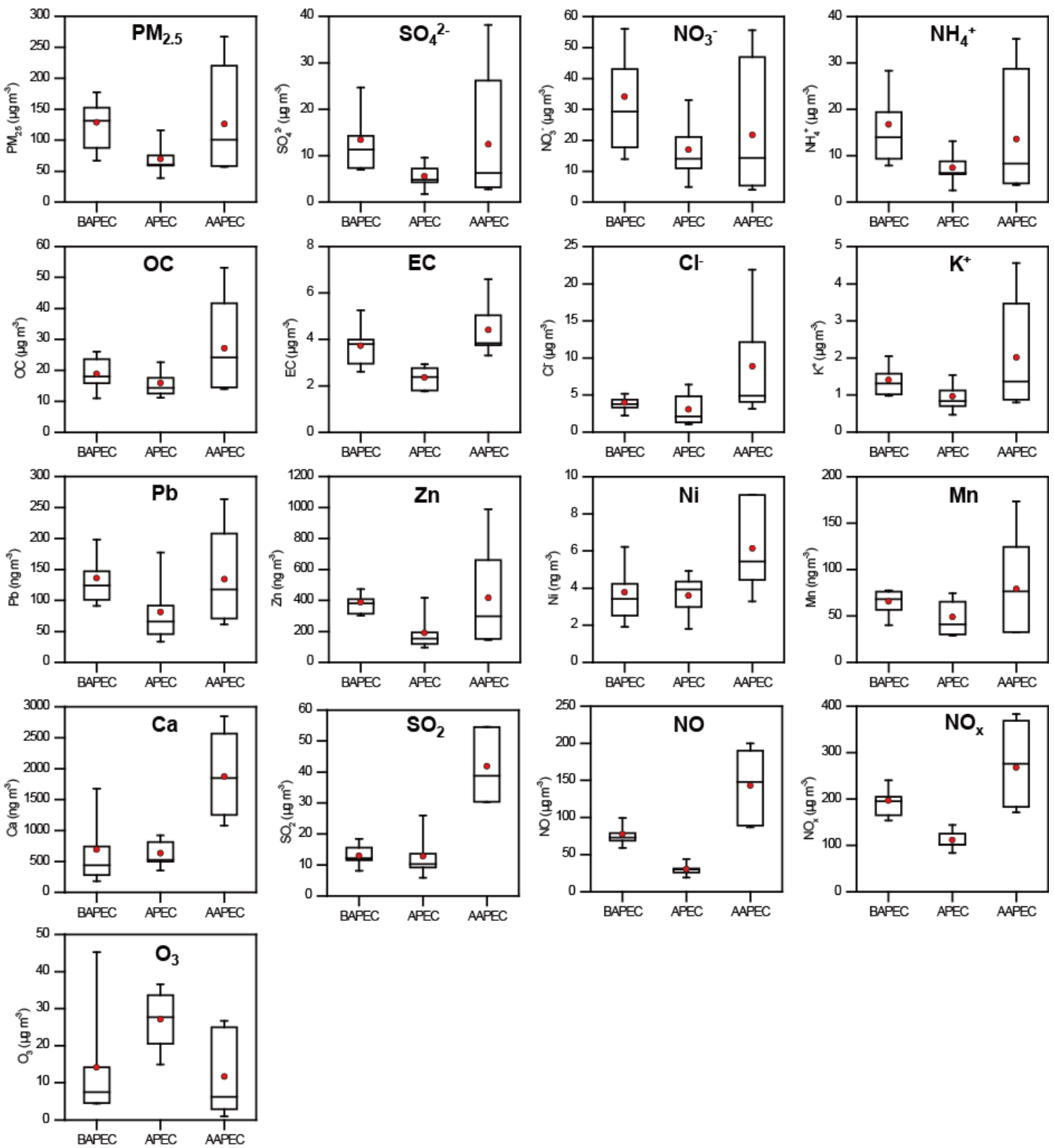

Figure S8. Variations of air pollutant concentrations during days with stable meteorological conditions during the APEC 2014 campaign, including $\mathrm{PM}_{2.5}$, SNA (sulphate + nitrate + ammonium), organic carbon (OC), elemental carbon (EC), $\mathrm{Cl}^{-}, \mathrm{K}^{+}$, elements $(\mathrm{Pb}, \mathrm{Zn}, \mathrm{Ni}, \mathrm{Mn}$, and $\mathrm{Ca})$, and gaseous pollutants $\left(\mathrm{SO}_{2}, \mathrm{NO}, \mathrm{NO}_{\mathrm{x}}\right.$, and $\left.\mathrm{O}_{3}\right)$. The red points represent mean values. The black cross bars are median values. The black box denotes the $25^{\text {th }}$ and $75^{\text {th }}$ percentiles. The whiskers represent the maximum and minimum, respectively. BAPEC/BParade: before APEC/Parade, AAPEC/AParade: after APEC/Parade. 

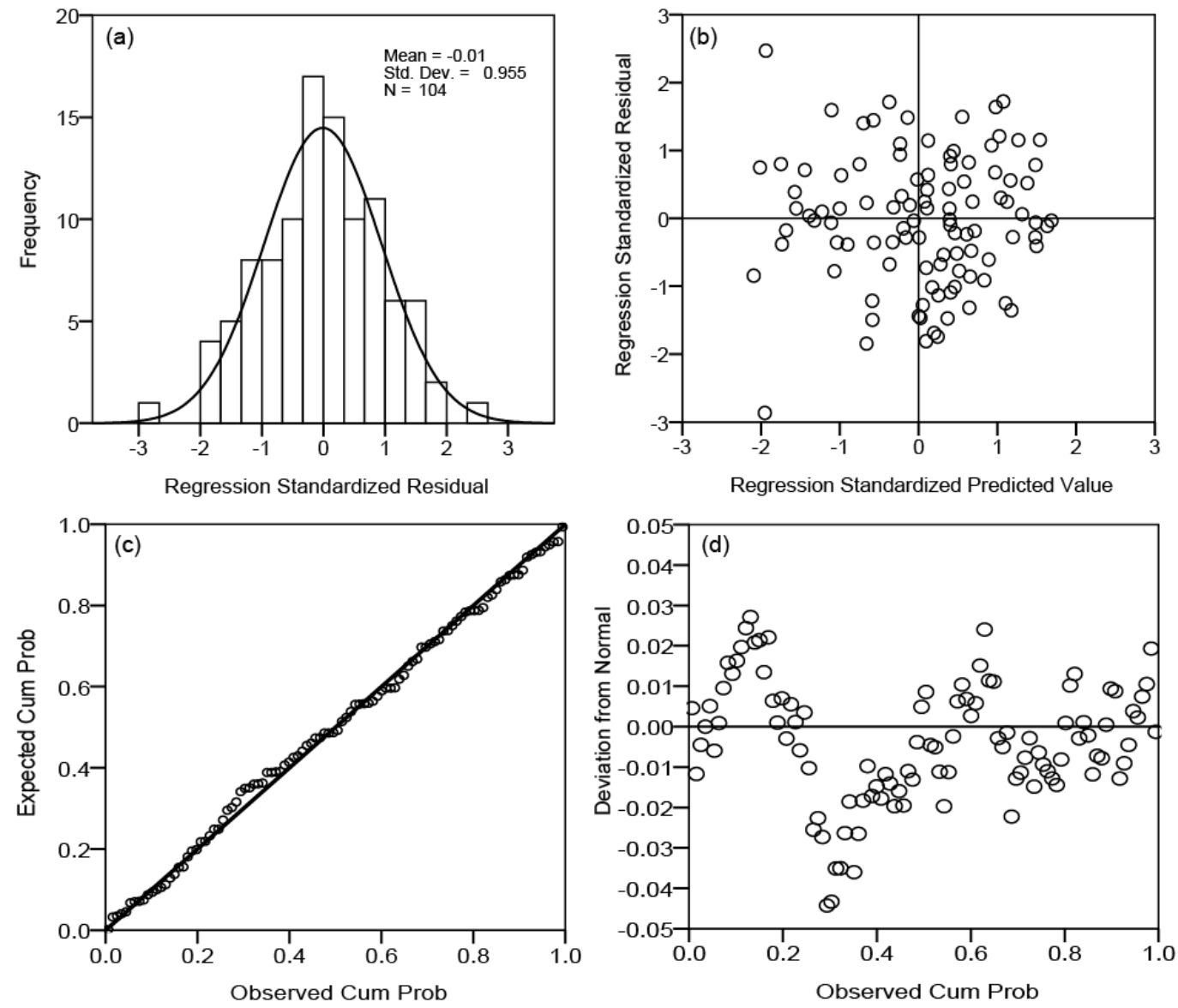

Figure S9. Residual analysis of the model. (a) Histogram of the regression standardized residual. (b) Scatter plot between the regression standardized predicted value and regression standardized residual. (c) Normal P-P plot of the regression standardized residual between the observed cumulative probability and expected cumulative probability. (d) De-trended normal P-P plot of the standardized residual of observed cumulative probability.

We used the $\mathrm{PM}_{2.5}$ model as an example. Figure $\mathrm{S} 9$ shows a residual analysis of the model. According to the residual histogram (a), the mean value of the regression standardized residual was -0.01 , with a standard deviation of 0.955 . According to the P-P graph (c), the distribution of the observed and expected cumulative probability spread along the diagonal of $y=x$. According to the de-trended P-P graph (d), the deviations from a normal distribution were within \pm 0.05 . These results indicate that the 
137 simulated values (b) could be applied to test the homoscedasticity, i.e. the distribution

138 of the regression residual did not change over the range of values predicted by the 139 regression.

140 
142 Table S10. Hypothesis K-S test summary of the unstandardized/standardized residual.

\begin{tabular}{|c|c|c|c|c|}
\hline & Null Hypothesis & Test & Sig.* & Decision \\
\hline 1 & $\begin{array}{l}\text { The distribution of Unstandardized } \\
\text { Residual is normal with mean - } \\
0.00 \text { and standard deviation } 0.40 \text {. }\end{array}$ & $\begin{array}{l}\text { One-Sample } \\
\text { Kolmogorov- }\end{array}$ & 0.96 & $\begin{array}{l}\text { Retain the null } \\
\text { hypothesis. }\end{array}$ \\
\hline 2 & $\begin{array}{l}\text { The distribution of Standardized } \\
\text { Residual is normal with mean - } \\
0.01 \text { and standard deviation } 0.96 \text {. }\end{array}$ & Smirnov Test & 0.96 & $\begin{array}{l}\text { Retain the null } \\
\text { hypothesis. }\end{array}$ \\
\hline
\end{tabular}

*The significance level is 0.05 .

143 the normal distribution. values. In this case, the test retains the null hypothesis that the distribution of unstandardized and standardized residual is normal, indicating that the residual follows 